$\xi=$

\title{
A brief exploration of fog computing architecture, technologies and challenges
}

\author{
Ramaiah Challa $^{1 *}$, K. Kiran Kumar ${ }^{2}$ \\ ${ }^{1}$ Department of $C S E, K L E F$, Vaddeswaram, $A P$ \\ ${ }^{2}$ Department of ECSE, KLEF, Vaddeswaram, AP \\ *E-mail: ramkluniversity@gmail.com
}

\begin{abstract}
Now a day's IoT systems are being used in rapid rate, so much data is being generated by massive ubiquitous things handling of that much data is not a simple issue it very critical task. Then again, despite the fact that that distributed computing has filled in as an efficient approach to process and store these information, in any case, challenges, for example, the expanding requests of ongoing or dormancy delicate applications and the impediment of system data transfer capacity, still can't be tackled by utilizing just cloud computing. Accordingly, another computing known as fog computing was proposed as extension of cloud computing. It brings the cloud services that are communication, computation and storage near to edge devices and users so latency can be reduced. In this papers details of fog computing are discussed.
\end{abstract}

Keywords: Cloud computing, Cyber-Physical System, Fog computing, IoT, Latency

\section{Introduction}

Now days we can observe that so many advancements are happening in the technologies. The technology made the usage of smart devices common. Internet of Things (IoT) applications is also used in rapid rate. In such kind of applications due to the usage of different devices, things so much data is going to be generated. Cyber-Physical System (CPS) as well as Mobile internet also generate huge amount of data.

As per the surveys by 2020 ending there will be more than 50 billion devices which are being associated with internet so that the data will be reaching to 500 zeta bytes. The immense information volume result in that the present handling and capacity abilities can't meet the demands. What's more, it is hard to be dealt with by conventional processing models, for example, dispersed registering, and distributed computing, and so on.

Since huge amount of data is there to be processed, cloud computing is chosen to process this much data as it possess high storage and computing powers. The cloud computing is a centralized model so most of the computation work is going to be done in the cloud [1]. For this the required data and requests are going to be shifted to centralized cloud. In spite of the fact that the information preparing speed has risen quickly, the system data transmission has not expanded considerably. So the network transfer speed is turning into the bottleneck of distributed computing for such an enormous measure of information. This may bring latency problems. Apart from the latency there is one more challenge that is processing is need to be done where there is no data center for cloud computing. In order to $t$ tackle these issues Fog Computing was proposed. The Fog computing can be successfully meet the requests of ongoing or inactivity delicate applications, and quite ease organize data transfer capacity bottlenecks.
Fog computing is suitable for dealing with an exceedingly dispersed and potentially visualized condition that gives process and system benefits among sensors and cloud server farms

\section{Literature Survey}

Saad Khan et. al., introduced an overview on existing writing on Fog processing applications to recognize normal security holes. Comparative innovations like Edge registering, Cloudlets and Micro-server farms have likewise been incorporated to give an all encompassing survey process. The dominant part of Fog applications are inspired by the want for usefulness and end-client necessities, while the security viewpoints are regularly disregarded or considered as an untimely idea. This paper likewise decides the effect of those security issues and conceivable arrangements, giving future security-pertinent headings to those in charge of planning, creating, and keeping up Fog frame works[3].

Carla Mouradian et. al., displayed an extensive study on haze processing. It basically audits the best in class in the light of a succinct arrangement of assessment criteria. We cover both the structures and the calculations that make haze frameworks. Difficulties and research headings are likewise presented[4]. What's more, the lessons realized are investigated and the prospects are talked about regarding the key part fog is probably going to play in rising innovations, for example, Tactile Internet.

Terms like, mobile cloud computing, mobile edge computing which are similar to fog computing are defined as follows Shanhe Yiet.al., [3]:

Local Cloud: A cloud which is built in a local network is called as local cloud that contains cloud-enabling software which runs on local servers and interacts with remote cloud. To improve the data 
privacy control, local cloud plays important role by executing cloud services on remote cloud.

Cloudlet: It is a job which is submitted to cloud; basically it has three parameters, job length, job type and time[8]. Cloudlet can be viewed as a "datacenter in a box" whose goal is to bring cloud closer. Cloudlet possesses sufficient computing power (RAM, CPU, etc.)

Mobile Edge Computing: Cloudlet and Mobile Edge Computing are having very similar characteristics[10]. But primarylocation (mobile base stations) of mobile edge computing made a small difference.

Mobile Cloud Computing: In Mobile Cloud Computing (MCC) infrastructure storage of data and processing of data happen externally from mobile devices by exporting computations to cloud. This tends the evolution of mobile edge computing.

Fog Computing: The non-trivial extension of cloud computing is fog computing[9]. The cloud computing technologies and challenges will shape in fog computing by emphasizing prominent properties like, broad network access, heterogeneity, geographical distribution, sand-boxed environment and flexible interoperability, and large scale of nodes.

\section{Architecture}

Fog computing is another computational worldview, which broadens the traditional distributed computing and administrations to the edge of system. It gives the calculation, correspondence, controlling, stockpiling and administrations capacities at the edge of system. The decentralized stage is unique in relation to other conventional computational model in design [7].

There are three layers present in the below architecture. They are

1. Terminal layer.

2. Fog layer.

3. Cloud layer.

Terminal layer is the layer nearest to the end client and physical condition. It comprises of different IoT devices, for instance, sensors, cell phones, shrewd vehicles, brilliant cards, per users, et cetera. These gadgets are broadly topographically circulated all in all. They are in charge of detecting the component information of physical articles or occasions and transmitting this detected information to upper layer for handling and storing.

Fog layer is situated on the edge of the system. Fog computing layer is made out of countless hubs, which for the most part including switches, portals, switchers, get to focuses, base stations, specific servers, and so forth. These fog nodes are broadly conveyed between the end gadgets and cloud. The end devise can helpfully associate with fog nodes to acquire administrations. They have the abilities to figure, transmit and incidentally store the received information. So that latency sensitive applications can be processed in fog layer.

The cloud computing layer is having of multiple high performance servers and storage devices, to provide various application services, such as smart home, smart transportation, smart grid, etc. It is having powerful computing and storage capabilities to support for extensive computation analysis and permanently storage of an huge amount of data. However, it is different from traditional cloud computing architecture, not all computing and storage tasks go through the cloud.

According to the demand load, the cloud core modules are efficiently managed and scheduled by some control strategies to improve utilization of the cloud resources

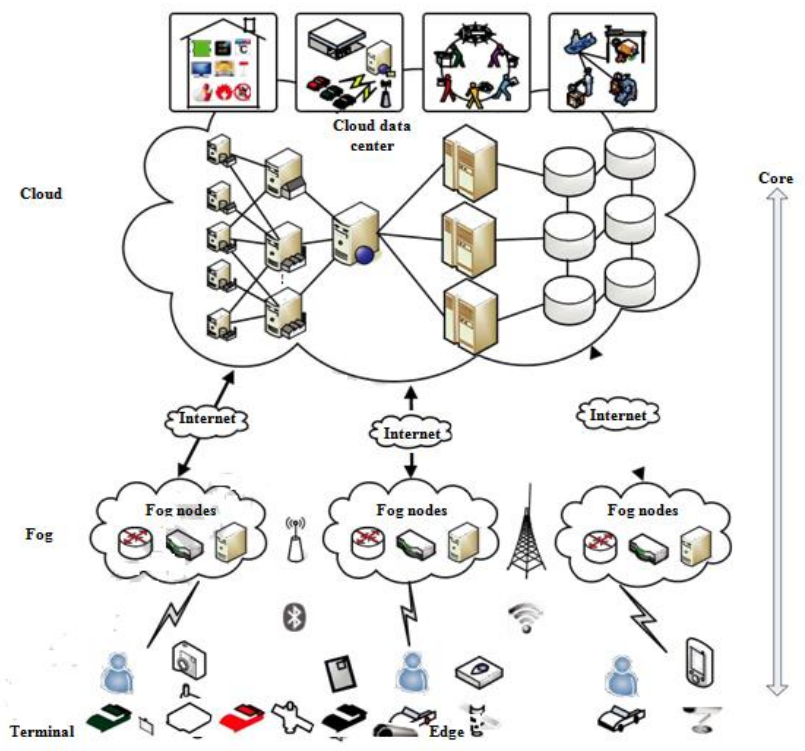

Fig. 1: Hierarchical structure of fog computing [1].

\section{Technologies required for Fog Computing}

Fog Computing can provide intelligent services and more adaptive services to users by depending on the requirements from applications. However some of them are some are already existing and common.

Here the technologies are categorized into three groups.

\subsection{Computing technologies:}

Fog nodes can serve local computation and data processing independently as well as autonomously. Capability of dealing low latency application needs to be supported by these computing technologies. The popular computing technologies are: Computation offloading, Latency management.

\subsection{Communication technologies:}

In fog computing model the Fog node plays very important role as intermediate networking component to connect cloud, fog nodes, deives and end users. It has three types of communications that are 1. The communication between fog nodes and centralized cloud data center can be wired or wireless. 2. Communication among the fog nodes can be either wired or wireless. 3. Usually the communication between fog nodes and end devices is wireless. 3G, 4G,WiFi, Wireless Local Area Networks (WLAN), ZigBee and Bluetooth are included in wireless communication protocols that they support the fog application. There are other communication protocols that are Software Defined Networking (SDN), Network Function Virtualization (NFA), 5G Wireless Communication System, Long Reach Passive Optical System (LRPOS), Content Distribution Network (CDN).

\subsection{Storage technologies:}

Fog computing is well suitable for low latency applications. In order to meet that the Pre-Cache Technology is considered. Fog nodes will forecast the user demand then actively select the required contents to the cache in distributed node so that required time to down load the data from remote data center can be reduced. By using storage resources efficiently fog applications provide desirable services. Since storage capabilities of fog applications are limited they use Storage Expansion Technology to improve overall service capability. 


\section{Challenges}

\subsection{Resource management:}

Finding the resources after that sharing them is very critical for application performance. In Fog computing paradigm, sharing of different kind of resources and all supported services among the central data centers, all mobile devices.

\subsection{Application-aware provisioning:}

Fog computing paradigm is needed to prepare and provide resources as the providing service mobility is the main criteria. The service mobility for end nodes is a challenge, because that metrics such as bandwidth, storage, computation and latency are going to be changed dynamically.

\subsection{Privacy and Security challenges:}

In Fog computing the following security challenges need to be handled:

Authentication

Access control.

Man in the middle.

Distributed denial of service.

\subsection{Programming platform:}

Since heterogeneous devices are going to be used by different programming languages programming platform became challenge.

\section{Conclusion}

This paper presented definition of Fog computing, it importance of Fog computing. And architecture of Fog computing with three layers has been presented. Important technologies for the Fog computing have been explored finally the challenges that are need to be addressed also presented. Here we can understand that if fog will come for full pledged implementation then it will avoid the delay problems of latency sensitive applications.

\section{References}

[1] Hu, P., Dhelim, S., Ning, H., \& Qiu, T. (2017). Survey on fog computing: architecture, key technologies, applications and open issues. Journal of Network and Computer Applications.

[2] Yi, S., Hao, Z., Qin, Z., \& Li, Q. (2015, November). Fog computing: Platform and applications. In Hot Topics in Web System and Technologies (HotWeb), 2015 Third IEEE Workshop on (pp. 73-78). IEEE.

[3] Khan, S., Parkinson, S., \& Qin, Y. (2017). Fog computing security: a review of current applications and security solutions. Journal of Cloud Computing, 6(1), 19.

[4] Mouradian, C., Naboulsi, D., Yangui, S., Glitho, R. H., Morrow, M. J., \& Polakos, P. A. (2017). A Comprehensive Survey on Fog Computing: State-of-the-art and Research Challenges. IEEE Communications Surveys \& Tutorials

[5] Mao, Y., You, C., Zhang, J., Huang, K., \& Letaief, K. B. (2017). A survey on mobile edge computing: The communication perspective. IEEE Communications Surveys \& Tutorials, 19(4) 2322-2358.

[6] J. Shi, J. Wan, H. Yan, H. Suo, A survey of cyber-physical systems, in: 2011 International Conference on Wireless Communications and Signal Processing (WCSP), 2011, pp. $1\{6$

[7] S. Yi, Z. Qin, and Q. Li, "Security and privacy issues of fog computing: A survey," in International Conference on Wireless Algorithms, Systems and Applications (WASA), 2015.

[8] M. Satyanarayanan, P. Bahl, R. Caceres, and N. Davies, "The case for vm-based cloudlets in mobile computing," Pervasive Computing, 2009
[9] F. Bonomi, R. Milito, J. Zhu, and S. Addepalli, "Fog computing and its role in the internet of things," in workshop on Mobile cloud computing. ACM, 2012.

[10] ETSI, "Mobile-edge computing," http://goo.gl/7NwTLE, 2014, [Online; accessed 18-June-2015]. 